Research Article

\title{
Involving community health workers in disease-specific interventions: perspectives from The Gambia on the impact of this approach
}

\author{
Ashley Preston', Joseph Okebe², Julie Balen³, Joan M Ribera ${ }^{4}$, Yoriko Masunaga ${ }^{5}$, Amadou Bah², Edgard Dabira², \\ Umberto D'Alessandro ${ }^{2}$ \\ ${ }^{1}$ SCI Foundation, London, UK, ${ }^{2}$ Disease Control and Elimination, Medical Research Council Unit, Fajara, The Gambia, 3 School of Health and Related \\ Research (ScHARR), The University of Sheffield, Sheffield, UK, ${ }^{4}$ PASS Suisse, Neuchâtel, Switzerland, 5 Faculty of Social and Behavioural Science, \\ Department of Sociology and Anthropology, University of Amsterdam, Amsterdam, The Netherlands and Institute of Tropical Medicine, Antwerp, \\ Belgium \\ Keywords: gambia, community health workers, global health \\ https://doi.org/10.29392/joghr.3.e2019084
}

\section{Journal of Global Health Reports}

Vol. 3, 2019

\begin{abstract}
Background
The Community Health Worker (CHW) programme is recognised as key for providing healthcare to communities, particularly in remote locations. CHWs are usually volunteers, nominated by their communities and trained to provide basic care and prevention for common illnesses. However, differences in disease-specific programmes aimed at meeting national agenda and perceived health needs of the community raises questions about the best approach to maximise the potential of this workforce.
\end{abstract}

\section{Methods}

This was an explorative qualitative study, ancillary to a larger trial on a malaria control intervention. In July 2017, 40 semi-structured interviews were conducted with 17 village health workers (VHWs), four community health nurses who supervise VHWs, and 19 key informants from the community. Analysis was concurrent to data collection and carried out using a deductive process for thematic analysis, with the aid of NVivo 11 Qualitative Analysis Software.

\section{Results}

There were three key aspects of the VHW role identified in this setting; (1) to give health advice; (2) to treat and refer patients; and (3) to support environmental cleaning. The VHWs' involvement in the clinical trial impacted their role in several ways. Overall, this was perceived very positively by the community and the VHWs since it improved access to medication and training on how to treat malaria. However, involvement was also perceived to increase VHWs' workload, and placed more emphasis on malaria over other common illnesses, creating a shift in the balance of their role between disease prevention and treatment.

\section{Conclusions}

VHWs are essential for the successful delivery of disease-specific activities at the community level. However, involving them in these activities has important implications for their everyday role. If carefully managed, it has the potential to improve their capacity to screen and treat specific diseases such as malaria.

The World Health Organization (WHO) recognises that the world is facing a chronic shortage of healthcare professionals, and its negative impact is felt more acutely in low and middle income countries, including Sub-Saharan African countries. ${ }^{1}$ In response to this crisis, one of the solutions recommended by WHO was to utilise community health workers (CHWs), volunteers selected from the community and trained to provide primary health care (PHC), to reach remote areas. ${ }^{2-4} \mathrm{CHWs}$ were first identified as key components of PHC and achieving the "Health for All" principle during the Alma-Ata conference, organised by the WHO in $1978 .{ }^{5}$ Key points from this meeting were the statement that health is a basic human right, inequality in health is unacceptable and that PHC is essential and should bring healthcare to the community level. ${ }^{6}$ Since the conference, CHW programmes have been implemented in many countries and there is evidence suggesting that $\mathrm{CHWs}$ contribute to the reduction of morbidity and mortality caused by dif- 
ferent diseases. ${ }^{7-9}$ For example, a Cochrane review concluded that interventions undertaken at the community level could improve timely access to malaria treatment and reduce childhood mortality. ${ }^{9}$ Trained CHWs also provide a cost-effective means of offering quality healthcare services in hard to reach, rural locations. $4,10,11$

Universal health coverage (UHC) is closely linked to the principles agreed at the Alma-Ata conference, aiming to provide high quality, equitable health services for all. Furthermore, UHC is recognised as a key platform to continue strengthening PHC services in order to achieve the Sustainable Development Goal of improved health for all. ${ }^{12,13}$ CHWs are a key aspect of the health workforce which could be strengthened, particularly as their initial purpose was to provide comprehensive, horizontal health services for the most common illnesses. Their role in helping implement disease-specific (vertical) programmes at community level has also been increasingly recognised. ${ }^{8,14-16} \mathrm{CHW}$ involvement in these programmes can increase their capacity to target specific diseases but can also distract from their original (broader) role. Where these programmes are not integrated into the national health system, the goals and expectations from the formal health system and the recipient communities risk becoming misaligned. ${ }^{17}$ Given that the success of CHW programmes depends on community participation and support $2,8,18$, it is important to understand the direction and implications of such a misalignment.

Despite the frequent engagement of CHWs in community-based disease control programmes, it is unclear how, and to what extent, involving them in such programmes impacts their everyday role or how they provide PHC service. ${ }^{19-24}$ Therefore, it is essential to improve our understanding of how CHWs progress national goals for disease control by implementing programmes at community level, whilst meeting the more general health needs of the community. This relationship was explored in the context of a community-based cluster randomised trial (hereafter referred to as RHOST) on reactive treatment of household contacts of clinically confirmed malaria cases in The Gambia. With significant reductions in the burden of malaria, The Gambia is working towards elimination and the trial was designed to evaluate reactive treatment as a strategy to reduce the human reservoir of transmission. ${ }^{25,26}$ Briefly, in the intervention villages all individuals living in the same compound of a clinical malaria case identified at health facilities or by the CHW (referred to as Village Health Workers (VHWs) in The Gambia) were systematically treated with dihydroartemisinin-piperaquine. VHWs were integral to the successful implementation of the trial at community level. During the trial, VHWs in the intervention arm were invited to undertake trial activities in addition to their routine roles. ${ }^{26,27}$ This study explores the role and everyday activities of VHWs and how these were balanced with and supported (or not) by the introduction of disease-specific activities relating to "vertical" programmes and interventions.

\section{METHODS}

\section{ETHICAL CONSIDERATIONS}

The project was approved by the London School of Hygiene and Tropical Medicine MSc Research Ethics Committee (reference: 14028, approved 21/06/17) and The Gambia Government/MRC Joint Ethics Committee (reference: SCC $1547 \mathrm{v} 1.1$, approved 15/06/17).

Prior to all interviews, the study was explained to participants in their preferred language. Written or thumb-print signatures; according to the literacy levels of each participant, were obtained as consent for participation and recording of interviews. Care was taken to protect the confidentiality of participants' identities and the data provided by only using password-protected devices to save data and using an encrypted recording device (DS-3500 Digital Voice Recorder, Olympus, Japan).

\section{STUDY DESIGN}

This was a qualitative study, ancillary to the cluster randomised control trial RHOST. Details of the trial design are reported elsewhere ${ }^{26,28}$ with results forthcoming. Briefly, RHOST comprises a complex intervention co-designed through multidisciplinary formative research, with direct involvement of the study communities and local health system actors in its implementation. The trial systematically targeted asymptomatic malaria-infected individuals, with the aim of reducing transmission; it also aimed to understand ways of effectively integrating the intervention into the existing health system structures. ${ }^{29}$ In the intervention arm of the trial, VHWs received training on their roles and responsibilities within the study, which included notifying the study team of clinical malaria cases in their village, treating all members of the case's compound with antimalarials and retrieving empty and unused drug sachets. In the control arm, index malaria cases were treated as usual (although cases were reported to the study team) and case's compound members were not treated. ${ }^{26}$

VHWs and identified key informants had already been exposed to key trial information and activities as part of the formative research and the "test phase" of the trial (August - December 2016). ${ }^{26}$ Data in this ancillary study were collected through semi-structured interviews with VHWs, community health nurses (CHNs) who supervise VHWs and key informants from the community. The interviews were conducted in July 2017, prior to the second phase of the trial (implementation).

\section{STUDY SETTING AND POPULATION}

The trial covered 34 villages in Central and Upper Baddibu, North Bank East Region (Figure 1), over three malaria transmission seasons (August - December 2016, 2017 and 2018). The area is rural and the population is predominantly engaged in farming. ${ }^{30}$ The main ethnic groups in the country, Wolof, Mandinka and Fula, are represented. Within villages, families usually live in large compounds containing multiple households. ${ }^{30}$ The community structure includes a village head, known as the Alkalo, and other important groups such as the Village Development Committee (VDC), 
Women's groups and Youth groups, which are involved in local administration. VHWs, traditional birth attendants (TBAs), and traditional healers who play important roles in the community's health and social activities.

\section{SAMPLING}

Initial sampling of villages that had been enrolled in the RHOST trial was done purposively; the main criteria included having a resident VHW and at least one malaria case reported in the test phase of the trial. The study team gained informed permission from the Alkalo before any interviews took place.

The sampling of participants was an ongoing, purposeful process based on the evaluation of emerging findings. All VHWs in the sampled villages were invited to participate in this study. Additionally, other key informants in the communities were invited, based on their exposure to trial activities; for example, attendance at organised community sensitisations for RHOST. Key informants were individuals who were important in the community structure; identified in a stakeholder analysis for RHOST as individuals in the community with the greatest influence to spread key RHOST messages. ${ }^{27} \mathrm{CHNs}$ were selected given their indepth knowledge of the VHW role and their link to the formal health system in The Gambia. Only adults (>18 years old) were invited to partake in this study.

\section{DATA COLLECTION}

Separate semi-structured interview guides were designed for VHWs, CHNs and other key informants. All interview guides followed the same format; starting with a set of general questions to determine demographics and followed by specific questions related to the research topic. These were piloted to ensure local acceptability and clarity. After every interview a summary sheet was written up immediately. It included the date, time and length of the interview, the location, who was being interviewed and who else was present, the language (if not English), a summary of the key points, any issues which occurred, and any initial observations. Summary sheets were used in the ongoing analysis of the data, allowing adaption of the interview technique or guides as needed.

Interviews took place in a location of the participant's choosing, usually in their house or compound, to ensure that they felt most at ease. Interviews were conducted in the participants' preferred language with the aid of a translator who had received training relating to the study aims, objectives and data collection tools and approaches. Interviews were recorded if informed consent was given; otherwise only notes were taken.

\section{DATA MANAGEMENT AND ANALYSIS}

Transcription and analysis were ongoing processes throughout the study. For interviews conducted in the local languages, only the sections spoken in English were transcribed. A deductive process for thematic analysis was used allowing themes to be identified and studied in detail from the beginning of the analysis. ${ }^{32}$ Two analytical frameworks

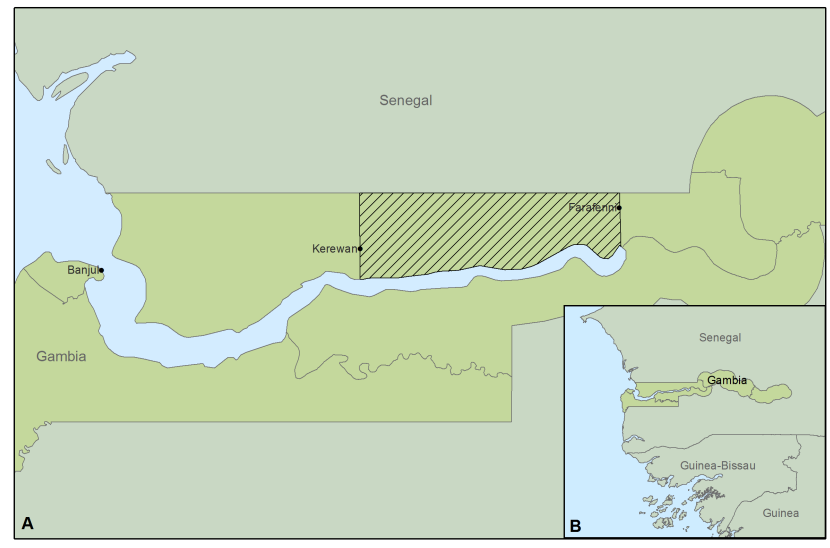

\section{Figure 1}

Map of Western Gambia. (A, B) The RHOST trial study site is situated north of the River Gambia, between Kerewan and Farafenni (as highlighted on the map) 31.

were designed, one for the professional perspective and one for the lay perspective. These were initially created based on the study objectives and heavily informed by the interview summary sheets. They included overarching themes and the codes relating to them. The frameworks were applied to the transcripts using NVivo 11 Qualitative Analysis Software (QSR International Pty Ltd. Cardigan UK). Coding was a flexible process, allowing the framework to be adapted in a data driven manner as new information emerged. ${ }^{32}$ The data was interpreted by consolidating the themes across all coded transcripts and triangulating information from three different perspectives (CHN, VHW and community).

\section{RESULTS}

\section{PARTICIPANT INFORMATION}

In total, 40 interviews with VHWs, CHNs and community members were conducted (Table 1).

Participants included VHWs from 17 of the 34 RHOST study villages; all were male, with an average age of 46 years (range: 30-65 years). Their primary occupation was farming, and they had also been working as VHWs usually for several years (ranging from two to over 10 years). All CHNs were male with an average age of 28 years (range: 25-35 years) and had been working as CHNs for an average of 2.5 years (range: 1.5-6 years). Male community members interviewed included village Alkalos, VDC chairmen, Youth Group leaders and one traditional healer; all of whom were farmers and had an average age of 60 years (range: 35-90 years). Female community members interviewed included TBAs and key members of the Women's Group; they were also involved in farming, with an average age of 40 years (range: 30-55 years).

\section{PERCEPTIONS OF THE VHW ROLE}

The key aspects of the VHW role identified by all participants were imparting health advice, treating and referring patients, and maintenance and cleaning of the local en- 
Table 1. Overview of respondents in this study.

\begin{tabular}{|c|c|c|c|c|}
\hline \multirow[t]{2}{*}{ Participants } & \multicolumn{4}{|c|}{ Study arm (intervention or control) } \\
\hline & Intervention only & Control only & Intervention or control & Total \\
\hline VHWs & 9 & 8 & N/A & 17 \\
\hline CHNs* & N/A & N/A & 4 & 4 \\
\hline Community Members & 10 (across 3 villages) & 9 (across 3 villages) & N/A & 19 \\
\hline Total & 19 & 17 & 4 & 40 \\
\hline
\end{tabular}

VHW - village health workers

*Community health workers; they work across intervention and control villages

vironment. VHWs reported performing weekly home visits where they held brief "health talks" with the residents, commonly about cleaning the village or how to prevent diarrhoea or malaria. VHWs were also involved in organising care and cleaning of the environment at the village level, with most having reported performing this at least once a month. Cleaning involved removing litter to designated village "dump sites" and filling in potholes to avoid "stagnant waters". VHWs and CHNs claimed that VHWs were trained to treat a small number of illnesses including malaria, diarrhoea, pneumonia (although the latter was only mentioned by CHNs), fever, body pain (such as headaches, stomach aches and chest pain) and small wounds; they referred more serious cases to the nearest health facility. Despite this, VHWs perceived their main role to be prevention rather than treatment of illnesses:

"I am not a doctor and my role is more of disease prevention, information dissemination, than treatment." (VHW, control village).

This sentiment was also emphasised by all four CHNs.

VHWs and CHNs reported that they met at least once a month. The CHN collected records of all illnesses seen and treated in the month by the VHW (on average about 6 people per week). VHWs were responsible for buying and replenishing their own drugs supplies, though the CHNs occasionally provided assistance. VHWs received support and training from the national health system and CHNs; this was perceived to be good which in turn contributed to improving the quality of the VHWs' work. Community members acknowledged that the original agreement was to support the VHW, either with aid on their farms or in buying medication; however, the majority of participants 33,34 admitted that little assistance from the communities was actually provided. This was the case even though they also understood that VHWs did not receive payment and did not charge for their services (only for the treatments they provide, but no profit is made from this). Conversely, uptake, trust and support from the community for the VHWs' work increased over time and understanding of the importance of disease prevention methods increased. For example, one community member explains about their VHW:

\footnotetext{
“...he make the village cleaner now, because like before, before when [the VHW] call people to come to a meeting, they say that's a waste of time, so they don't attend to his meetings. But now it's not like that, even if say now he wants to see the village everybody will come..." (VDC member, intervention village).
}

| The perceived disease focus of the VHWs.

Despite the perception that malaria had been reducing in the area, there was a general consensus by interviewees that it was still the main focus of the VHW role. One VHW described his disease focus:

"When I started up till now, the most ailment I treat is malaria, that's the most one that I spend most time treating, than other [diseases]". (VHW, control village).

Most VHWs (16/17) also stated that malaria was the biggest health issue in the community, with one VHW commenting:

"Malaria is the most disease that is being complained of from the community, yeah that's the main one." (VHW, control village).

Suggested reasons for the large amount of time and energy spent on malaria included that VHWs still did a lot of prevention work and that treatment was perceived to take a long time in comparison to other illnesses. Furthermore, a small number of VHWs and community members perceived that the VHWs would continue to focus on malaria despite a reduction in disease burden because it was the focus of their training. One community member explained:

$$
\begin{aligned}
& \text { "[The VHW] has the materials to test malaria but for other } \\
& \text { ailments he might not have the knowledge or materials... } \\
& \text { But for malaria he can." (Youth Group Leader, interven- } \\
& \text { tion village). }
\end{aligned}
$$

Three VHWs admitted that if they had the expertise and medication to deal with diseases other than malaria, this would improve their ability to meet all the needs of the community. VHWs are only trained on the most common illnesses; therefore, if patients showed signs of complications or presented with a rare or more serious illness such as hypertension, they are referred, as one VHW explains:

\footnotetext{
"The ones I'm treating, I'm doing my best, but there are many other sicknesses that I refer to the nearest health facility, because I don't have the knowledge or the means to deal with those." (VHW, control village)
}

Community members from both intervention and control villages agreed that a wider focus than the most common illnesses already stated would be most beneficial for the community, especially as some felt that other conditions such as hypertension, were now more important than malaria. 
| Challenges faced by the VHWs.

A key concern voiced by multiple VHWs was that they did not have an appropriate place to store medication or consult patients. Several (8/17) also mentioned shortages of medical supplies, with one stating:

"The problems I'm facing is I don't have the necessary medication... and also I run, sometimes I run out of medication." (VHW, control village).

Problems of mobility were addressed by the provision of bicycles as part of a new "TB project”:

"I had a mobility problem and now I'm provided with a bike, even if I go out of the village or visit the farms, if I'm called I come back very quickly." (VHW, control village).

The workload was considered challenging by VHWs because they had to balance this role with their personal work, such as farming. Despite this, VHWs claimed to generally prioritise their role as a VHW above other tasks, often leaving their farms to attend to patients. This was confirmed by community members as VHW availability was reportedly excellent:

"[The VHW] is mostly found at the compound and there are cases where he is found at the farms, then the people call him if there is any patient at his place, and he will come." (TBA, intervention village).

Despite this, there was the sense that the workload had reduced over time, mainly due to a reduction in the burden of malaria and other diseases. As one community member explains:

"[The VHW's] load depends on the number of diseases that he treats, before he was concentrated on wide range of diseases and now most of those diseases are not seen, they are gone, so now his workload has gone less..." (VDC Chairman, control village).

In addition, VHWs felt they were better able to manage their work; for example:

"It was heavy for me before when I was starting but then I didn't get the required training... But as time goes on it was lighter because I had a lot of trainings and the people I was dealing with have confidence in me..." (VHW, control village).

\section{THE PERCEIVED IMPACTS OF RHOST ON THE VHW ROLE}

\section{POSITIONALITY OF THE RHOST PROIECT AND RESULTING PERCEPTIONS}

Due to its long history of research in the area, the Medical Research Council Unit in The Gambia (MRCG) occupies a unique niche; it is often perceived as a substitute for the national health system, as many people look to the MRCG for treatment of illnesses, usually in the context of research studies. As such, the position of the MRCG and specific projects such as RHOST were frequently indistinguishable by the communities. Furthermore, in the intervention villages $6 / 10$ community members interviewed mentioned the relationship between the VHW and the community had im- proved through the former's work with the MRCG, as a community member explained:

"[The VHW] has trust from the community, they trusted him very well and MRC[G]'s collaboration has earned him respect." (Women's Group President, intervention village)

Changes in the VHW role attributed to RHOST

Most community members (8/10) and VHWs (8/9) in the intervention villages claimed that RHOST had improved the VHWs' ability to treat malaria as a result of the trainings offered and improvements in the supply of medication and equipment, for example:

“The coming of the MRC[G] project has increased [the
VHW's] knowledge on how to treat. [The VHW] has [Rapid
Diagnostic Tests] to test people, before he hadn't those
things, he just assumed that you had malaria and giving
medication." (TBA, intervention village).

This was further emphasised by statements made by VHWs, for example:
"...the medication that [MRCG] gives and also the educa- tion that we get from the project, it helps us improve our work." (VHW, intervention village).

This perceived increase in support and training, was not necessarily beneficial to the VHWs as in some cases it contributed to a reduction in community support, as described by one VHW:

"...when [the community] see MRC[G] people around me... they will say that I'm given something. So they discuss that nobody will help me because I've been given something by $M R C[G]$ people." (VHW, intervention village).

The majority of the community members suggested that the project had increased their VHWs' workload, with some even suggesting it had helped the VHW focus more on their role. Commonly mentioned reasons were that, because of the improved ability of the VHW to treat malaria, the VHW could consequently treat more people, and that meeting RHOST personnel and the additional training took time. Surprisingly only one community member mentioned that the workload increased because the VHW was required to treat a greater number of people as part of the project; they explained:

"The amount of people [the VHW] has been treating has
increased on the side of malaria, because you have to give
[antimalarials] to, maybe if it is a big family 20 or 30 peo-
ple, so that's why there is an increase." (VDC Chairman,
intervention village).

The VHWs had differing perspectives on the impact of the project on their work schedule. One VHW agreed that communicating and the interactions required with RHOST personnel increased their workload, while another suggested that the trial had improved the VHWs' relationship with the community and this had increased demand for their services, as he explained: 
"As a result of the project, lot of people who were not coming to see me, they are now seeing me..." (VHW, intervention village).

However, others suggested that RHOST had led to a decrease in workload, because they were better able to handle their work due to the project training, and the project activities helped sensitise the community about malaria, making the VHWs' work easier. The most common reason given (mentioned by four VHWs) was linked to the project being perceived to cause a reduction in malaria transmission:

\section{"...if the case is treated all the people in the compound are also treated, so I hardly see another somebody infected with malaria in the same compound, compared to before when I treat the person, the next day I will see another person." (VHW, intervention village). \\ Involvement in RHOST Likely influenced the priorities of the VHW role.}

It seems that the focus of the VHWs may be further skewed towards malaria because of their involvement in RHOST: all but one VHW suggested that malaria was the biggest health issue, with one VHW even suggesting their focus on malaria had increased because the project had increased his workload. In contrast, community members and CHNs, who had less exposure to the key trial messages, were not as likely to mention malaria as a health issue. However, there was a small amount of evidence to the contrary; for example, when asked directly, three VHWs suggested the project had not influenced their focus on malaria and one VHW suggested that their focus had lessened since the project has led to reduced malaria cases in the community. Furthermore, whilst RHOST was only ever designed to target malaria, the distinction between trial and national health system was not clear at community level. Therefore, some community and VHWs suggested that they would appreciate the MRCG expanding to target additional diseases to meet all the needs in their community. For example, whilst the main focus of one VHW was on malaria, he also suggested:

\section{“...after finishing malaria if [MRCG] can step on another disease that disturb people that would be good, because especially $M R C[G]$ guys they told us that $M R C[G]$ have re- duced a lot concerning malaria." (VHW, intervention vil- lage).}

In particular, he said that hypertension needs attention because it also "disturbed people". This perceived disconnect between the trial aims and the perceived needs of the community was further emphasised by a community member who appealed for an expansion of the project to include hypertension:

"MRC[G] concentrates on the malaria, but if they can extend the project and involve other diseases like hypertension, it's a big problem to the community now... There are many diseases so they shouldn't concentrate on one disease alone." (Women's Group President, intervention village).

There is also evidence to suggest that the project influenced the VHWs' focus from prevention to treatment. Evidence comes from three VHWs in the control arm of the trial who strongly emphasised (without prompting) that their main role was for disease prevention. All CHNs, who had relatively little exposure to RHOST, also emphasised that the VHW role focused on prevention over treatment. However, the main focus of the role on prevention was not mentioned as explicitly for VHWs in the intervention arm.

\section{DISCUSSION}

In The Gambia, like in many countries, VHWs are integral to the country's PHC strategy because they form part of the community level health system in rural areas. They play a key role in disease prevention and treatment of common illnesses, and as such they are generally perceived favourably within their communities. ${ }^{27,35}$ However, in their day-to-day role, VHWs in The Gambia reportedly faced certain challenges, some of which were resolved through their involvement in RHOST; for example, the trial was said to have improved VHW's outputs through training and greater availability of resources. Their involvement in RHOST was therefore viewed positively, since it reportedly enhanced their capacity to treat malaria. While this may have been influenced in part by responder bias and the perceptions related to the long-standing presence of the MRCG in The Gambia, evidence suggests that before the trial shortages of drug supplies were common in this area, with marked improvements during the trial period. In comparison to routine health systems in low- and middle-income countries, short term projects like RHOST are better able to target funding for specific activities, thus supporting necessary supplies like medication. ${ }^{26,36}$ These improvements have been noted in other studies in The Gambia, for example, VHWs involved in administering seasonal malaria chemoprevention to children had improved access to antimalarials, oral rehydration solution, and paracetamol syrup compared to health facilities where stock-outs were common. ${ }^{23}$ Furthermore, within this study those VHWs involved in the "TB project" were provided a bicycle which alleviated the challenges they faced with travelling for their role. This suggests that the RHOST trial had the ability to increase VHWs' capacity, but these gains are unlikely to be sustainable beyond the duration of the project. ${ }^{23,37}$

Other reported impacts of RHOST on the VHW role included an increased workload and reduced support from the community. The perception by the community that VHWs received all necessary support from trial personnel was likely partly influenced by the long-standing presence of the MRCG in The Gambia, as the community often does not distinguish between the support provided by different short-term projects. However, similar situations have also been documented in other CHW programmes; for example, in Kenya, some community members did not believe that the CHWs were volunteers which led to tension and difficulties for the CHWs. ${ }^{38}$ Many studies across Sub-Saharan Africa have now recognised that $\mathrm{CHW}$ performance is intrinsically linked to the community context, with the success of their work depending on community backing and engagement. $2,8,18,39,40$ These experiences show that in the short-term, effective sensitisation which is adaptive to changing perceptions is essential. ${ }^{2,8,18}$ It also highlights that in the long-term, asking the local communities to sup- 
port CHWs for the work they provide is not realistic. CHWs should be an integral part of the health system and as such should be compensated for the work they provide.

Care also needs to be taken to appropriately manage the increased workload due to intervention activities, particularly as high workload was a pre-existing challenge reported by VHWs in this study, and in other settings. ${ }^{41}{ }^{34}$ Without careful management, the addition of trial activities could negatively affect the quality of their work. ${ }^{4,42}$ A study on the workload of CHWs in Malawi found that they were increasingly taking on activities related to treatment due to task shifting health services to CHWs. Subsequently, the CHWs were overloaded and felt that they were not adequately equipped to carry out their expanded role. ${ }^{34}$ However, a literature review assessing the ability of CHWs to take on extra tasks, such as the use of rapid diagnostic tests for malaria case management, concluded that with appropriate training and supervision, quality of care can be maintained. ${ }^{4}$ This highlights that in any context, if CHWs have the appropriate support to take on additional tasks, their increasingly comprehensive role will be highly beneficial to the community and for their effectiveness whilst engaged in disease-specific interventions. ${ }^{4,43-45}$ Therefore, acknowledging the complexity of the CHW role which is influenced by both the community and health system context, and understanding the existing challenges faced by CHWs in their everyday role, is essential to successfully engage them in disease-specific interventions. ${ }^{39,40,46}$

A key finding of this study was that involvement with RHOST may have increased the VHWs' focus on treatment whereas the original role of the VHW was reported to focus primarily on the prevention of diseases, as emphasised by multiple VHWs in the control villages and all CHNs. Caution must be taken when drawing this conclusion, as the data are not definitive and the role of the VHW will likely change over time as national health strategy changes. However, it is not uncommon for similar programmes to skew the priorities of CHWs given that they require the $\mathrm{CHW}$ to perform additional activities in relation to specific diseases. 37,39,47,48 For example, a study looking at HIV, TB and malaria control programmes in Papua New Guinea, found that the programmes increased the emphasis for the control and treatment of these diseases. ${ }^{37}$ This emphasises the importance of integration of disease-specific activities into the national health system to ensure that when CHWs are involved in additional projects or trials, this does not detract from their routine PHC activities and an appropriate balance between prevention and treatment is maintained. ${ }^{14,15,49}$ If integration does not occur, too much focus may be put on specific diseases and this can lead to over diagnosis and treatment. ${ }^{49,50}$ Furthermore, integration has been highlighted as a key way to improve CHW capacity for targeting multiple diseases. ${ }^{40,48}$ This is important in the face of changing disease dynamics, such as reducing malaria burden, to ensure the CHW system is flexible enough to meet the changing needs of the community. 4,48

At a national or global scale, VHW involvement in disease-specific programmes is beneficial for elimination efforts. Over the past decade or so there has been a renewed energy globally to control and eliminate malaria and RHOST is a key example of the research going into how best to target areas of low malaria burden. 5,51,52 The ethics and feasibility of elimination campaigns have been debated over the years, because when a disease nears elimination it is no longer a health burden and so may lose public support. ${ }^{53,54}$ VHWs in this study commonly emphasised the importance of specific diseases at the community level, thus highlighting how they could aid elimination efforts by helping to maintain support.

In 2012 the Gambian Ministry of Health and Social Welfare undertook a situational analysis to understand how a WHO malaria strategy, introduced in 2005, influenced the PHC strategy. They concluded that the malaria strategy was well-integrated, but that there was the risk that donor funding, which was more abundant for malaria than other common diseases, could adversely impact the PHC strategy by creating disease-specific VHWs. ${ }^{35}$ Evidence from this study suggests that VHW involvement in disease-specific trials could also exacerbate this issue, especially as some VHWs and community members perceived that the VHWs were only trained to deal with malaria. Therefore, although the current VHW focus may be in line with the country's malaria strategy, care needs to be taken to ensure VHW involvement with disease-specific activities is balanced with the other health needs of the community; thus maintaining alignment with the country's overarching PHC strategy. ${ }^{27}$

Some potential limitations of this study to note are that a small subset of the interviews (all ten community members from intervention villages) were conducted subsequent to the sensitisation for the second phase of RHOST, likely influencing community perceptions; although it may have reduced recall bias when discussing the impact of the project. To mitigate this, more effort was taken before these interviews to explain the purpose of the study and to emphasise that the aim included understanding the VHW in general, not just in relation to the project. Also, the interviews were conducted with a Gambian translator, fluent in English and the local languages; therefore, only the English sections of the interviews were transcribed as there was only a small risk of meanings being lost in translation.

\section{CONCLUSIONS}

In conclusion, VHWs play a key role in improving access to disease prevention messages and treatment in rural Gambia, and overall their role and their involvement with RHOST was perceived very favourably by community members. Their position in the community makes their involvement in disease-specific trials such as RHOST essential; however, this has had important implications for their everyday role. It helped improve their capacity to treat malaria, both with improved training and access to resources, but in doing so it has also skewed their focus towards malaria and treatment. Care needs to be taken that the balance between appropriate prevention and treatment activities is maintained, and to ensure positive changes caused by involvement in disease-specific projects are sustainable. 


\section{ACKNOWLEDGEMENTS}

The authors thank all study participants, namely the Village Health Workers, Community Health Nurses and key informants within the community, who gave their time and effort to be interviewed.

The project was approved by the London School of Hygiene and Tropical Medicine MSc Research Ethics Committee (reference: 14028, approved 21/06/17) and The Gambia Government/MRC Joint Ethics Committee (reference: SCC $1547 \mathrm{v} 1.1$, approved 15/06/17).

Prior to all interviews, the study was explained to participants in their preferred language. Written or thumb-print signatures were given as consent for participation and recording of interviews, taking into consideration the literacy levels of each participant. Care was taken to protect the confidentiality of participants' identities and the raw data by only using password-protected devices to save data and using an encrypted recording device (DS-3500 Digital Voice Recorder, Olympus, Japan).

\section{FUNDING}

Funding for this research was provided by the London School of Hygiene and Tropical Medicine, as part of the MSc
Control of Infectious Diseases course which provides a research bursary. The main RHOST project was funded by the Joint Global Health Trials Scheme (Medical Research Council (United Kingdom), Department for International Development (DFID), and Wellcome Trust). The funders had no role in the design or preparation of the manuscript.

\section{COMPETING INTERESTS}

The authors completed the Unified Competing Interest form at http://www.icmje.org/coi disclosure.pdf (available upon request from the corresponding author), and declare no conflicts of interest.

\section{CORRESPONDENCE TO:}

Ashley Preston; BSc Biology, MSci Biology, MSc Control of Infectious Diseases

Edinburgh House

170 Kennington Ln

Lambeth

London SE11 5DP

UK

a.preston@schisto.org.uk 


\section{REFERENCES}

1. WHO. Task Shifting: Rational Redistribution of Tasks Among Health Workforce Team - Global Recommendations and Guidelines. Published online 2008.

2. Lehmann U, Van Damme W, Barten F, Sanders D. Task shifting: the answer to the human resources crisis in Africa? Hum Resour Health. 2009;7(1):49. do i:10.1186/1478-4491-7-49

3. Baine SO, Kasangaki A. A scoping study on task shifting; the case of Uganda. BMC Health Serv Res. 2014;14(1):184. doi:10.1186/1472-6963-14-184

4. Smith Paintain L, Willey B, Kedenge S, et al. Community health workers and stand-alone or integrated case management of malaria: a systematic literature review. Am J Trop Med Hyg. 2014;91(3):461-470. doi:10.4269/aitmh.14-0094

5. Cueto M. The origins of primary health care and selective primary health care. Am J Public Health. 2004;94(11):1864-1874. doi:10.2105/ajph.94.11.1864

6. World Health Organisation. Declaration of AlmaAta. USSR; 1978.

7. Lewin S, Dick J, Pond P, et al. Lay health workers in primary and community health care. Lewin S, ed. Cochrane Database Syst Rev. Published online January 24, 2005. doi:10.1002/14651858.cd004015.pub2

8. Bhattacharyya K, Winch P, Leban K, Tien M. Community Health Worker Incentives and Disincentives: How They Affect Motivation, Retention, and Sustainability. Basic Support for Institutionalizing Child Survival Project (BASICS II), United States Agency for International Development; 2001.

9. Okwundu CI, Nagpal S, Musekiwa A, Sinclair D. Home- or community-based programmes for treating malaria. Okwundu CI, ed. Cochrane Database of Systematic Reviews. 2013;(5). doi:10.1002/14651858.c d009527.pub2

10. Liverani M, Nguon C, Sok R, et al. Improving access to health care amongst vulnerable populations: a qualitative study of village malaria workers in Kampot, Cambodia. BMC Health Serv Res. 2017;17(1):335. doi:10.1186/s12913-017-2282-4

11. Seidman G, Atun R. Does task shifting yield cost savings and improve efficiency for health systems? A systematic review of evidence from low-income and middle-income countries. Hum Resour Health. 2017;15(1):29. doi:10.1186/s12960-017-0200-9
12. UNDP. UNDP Support To The Implementation Of The 2030 Agenda For Sustainable Development. New York; 2016.

13. World Health Organisation. SDGs and Progress towards Universal Health Coverage. Regional Office for South East Asia: WHO; 2017.

14. Rule J, Ngo DA, Oanh TTM, et al. Strengthening Primary Health Care in Low- and Middle-Income Countries. Asia Pac J Public Health. 2013;26(4):339-348. doi:10.1177/1010539513503869

15. Jegede AS, Oshiname FO, Sanou AK, et al. Assessing Acceptability of a diagnostic and malaria treatment package delivered by community health workers in malaria-endemic settings of Burkina Faso, Nigeria, and Uganda. Clin Infect Dis. 2016;63(suppl 5):S306-S311. doi:10.1093/cid/ciw630

16. Mijovic H, McKnight J, English M. What does the literature tell us about health workers' experiences of task-shifting projects in sub-Saharan Africa? A systematic, qualitative review. J Clin Nurs. 2016;25(15-16):2083-2100. doi:10.1111/jocn.13349

17. Lehmann U, Sanders D. Community Health Workers: What do we know about them? Published online 2007.

18. Mathews C, van der Walt H, Barron P. A shotgun marriage - community health workers and government health services. Qualitative evaluation of a community health worker project in Khayelitsha. $S$ Afr Med J. 1994;Oct;84(10):659-63:659-663.

19. Alonso PL, Lindsay SW, Armstrong JRM, et al. The effect of insecticide-treated bed nets on mortality of Gambian children. Lancet. 1991;337(8756):1499-1502. doi:10.1016/0140-6736(91)93194-e

20. Greenwood B. Comparison of two strategies for control of malaria within a primary health care programme in The Gambia. Lancet. 1988;331(8595):1121-1127. doi:10.1016/s0140-6736(8 8) $91949-6$

21. Menon A, Snow RW, Byass P, Greenwood BM, Hayes RJ, N'jie ABH. Sustained protection against mortality and morbidity from malaria in rural Gambian children by chemoprophylaxis given by village health workers. Trans $R$ Soc Trop Med Hyg. 1990;84(6):768-772. doi:10.1016/0035-9203(90)9007 1-1 
22. Sesay S, Milligan P, Touray E, et al. A trial of intermittent preventive treatment and home-based management of malaria in a rural area of The Gambia. Malar J. 2011;10(1):2. doi:10.1186/1475-287 5-10-2

23. Bojang KA, Akor F, Conteh L, et al. Two strategies for the delivery of IPTc in an area of seasonal malaria transmission in the Gambia: a randomised controlled trial. PLoS Med. 2011;8(2):e1000409. doi:10.1371/jour nal.pmed.1000409

24. Scott S, Mens PF, Tinto H, et al. Communitybased scheduled screening and treatment of malaria in pregnancy for improved maternal and infant health in The Gambia, Burkina Faso and Benin: study protocol for a randomized controlled trial. Trials. 2014;15(1). doi:10.1186/1745-6215-15-340

25. Mwesigwa J, Okebe J, Affara M, et al. On-going malaria transmission in The Gambia despite high coverage of control interventions: a nationwide crosssectional survey. Malar J. 2015;14(1):314. doi:10.118 6/s12936-015-0829-6

26. Okebe J, Ribera JM, Balen J, et al. Reactive community-based self-administered treatment against residual malaria transmission: study protocol for a randomized controlled trial. Trials. 2018;19(1). $\underline{\mathrm{d}}$ oi:10.1186/s13063-018-2506-X

27. Ministry of Health and Social Welfare. National Health Policy 2012-2020: Acceleration of Quality Health Services and Universal Coverage. Fajara; 2012.

28. D’alessandro U, Okebe J. Clinical Trial Protocol: Reactive Household-Based Self-Administered Treatment against Residual Malaria Transmission: A Cluster Randomized Trial. Unpublished; 2017.

29. Jaiteh F, Masunaga Y, Okebe J, et al. Community perspectives on treating asymptomatic infections for malaria elimination in The Gambia. Malar J. 2019;18(1). doi:10.1186/s12936-019-2672-7

30. Muela J, Masunaga Y, Jaiteh F, Peeters K. Formative Research for RHOST First Report. Unpublished; 2016.

31. GAUL. World Map, GAUL Level 1. Published 2019. http://worldmap.harvard.edu/data/geonode:g2008_1

32. Braun V, Clarke V. Using thematic analysis in psychology. Qual Res Psychol. 2008;3(2):77-101. doi:1 $\underline{0.1191 / 1478088706 q p 0630 a}$

33. Seutloali T, Napoles L, Bam N. Community health workers in Lesotho: Experiences of health promotion activities. African J Prim Heal care Afr J Prim Health Care Fam Med. 2018;10:e1-8:1-8. doi:10.4102/phcf m.v10i1.1558
34. Smith S, Deveridge A, Berman J, et al. Taskshifting and prioritization: a situational analysis examining the role and experiences of community health workers in Malawi. Hum Resour Health. 2014;12(1):24. doi:10.1186/1478-4491-12-24

35. Ministry of Health and Social Welfare. Situational Analysis Home Management of Malaria in the Context of Primary Health Care in The Gambia. Fajara; 2012.

36. Korenromp EL, Hosseini M, Newman RD, Cibulskis RE. Progress towards malaria control targets in relation to national malaria programme funding. Malar J. 2013;12(1):18. doi:10.1186/1475-2875-12-18

37. Rudge JW, Phuanakoonon S, Nema KH, MounierJack S, Coker R. Critical interactions between Global Fund-supported programmes and health systems: a case study in Papua New Guinea. Health Policy Plan. 2010;25(Suppl. 1):i48-i52. doi:10.1093/heapol/czq058

38. Oliver M, Geniets A, Winters N, Rega I, Mbae SM. What do community health workers have to say about their work, and how can this inform improved programme design? A case study with CHWs within Kenya. Glob Health Action. 2015;8(1):27168. doi:10.34 $\underline{02 / \text { gha.v8.27168 }}$

39. Kok MC, Broerse JEW, Theobald S, Ormel H, Dieleman M, Taegtmeyer M. Performance of community health workers: situating their intermediary position within complex adaptive health systems. Hum Resour Health. 2017;15(1):59. doi:10.11 86/s12960-017-0234-z

40. Baynes C, Semu H, Baraka J, et al. An exploration of the feasibility, acceptability, and effectiveness of professional, multitasked community health workers in Tanzania. Global Public Health. 2017;12(8):1018-1032. doi:10.1080/17441692.2015.10 $\underline{80750}$

41. Gau YM, Buettner P, Usher K, Stewart L. Burden experienced by community health volunteers in Taiwan: a survey. BMC Public Health. 2013;13(1):491. doi:10.1186/1471-2458-13-491

42. Lin Y, Li L, Mi F, et al. Screening patients with Diabetes Mellitus for Tuberculosis in China. Trop Med Int Health. 2012;17(10):1302-1308. doi:10.1111/j.136 5-3156.2012.03069.x

43. Kruk ME, Porignon D, Rockers PC, Van Lerberghe W. The contribution of primary care to health and health systems in low- and middle-income countries: A critical review of major primary care initiatives. Soc Sci Med. 2010;70(6):904-911. doi:10.1016/i.socscime d.2009.11.025 
44. Sunguya BF, Mlunde LB, Ayer R, Jimba M. Towards eliminating malaria in high endemic countries: the roles of community health workers and related cadres and their challenges in integrated community case management for malaria: a systematic review. Malar J. 2017;16(1):10. doi:10.1186/s12936-016-1667-x

45. Gilmore B, McAuliffe E. Effectiveness of community health workers delivering preventive interventions for maternal and child health in lowand middle-income countries: a systematic review. BMC Public Health. 2013;13(1):847. doi:10.1186/147 1-2458-13-847

46. Kasteng F, Settumba S, Källander K, Vassall A. Valuing the work of unpaid community health workers and exploring the incentives to volunteering in rural Africa. Health Policy Plan.

2016;31(2):205-216. doi:10.1093/heapol/czv042

47. Shelley KD, Frumence G, Mpembeni R, et al. Can volunteer community health workers manage multiple roles? An interrupted time-series analysis of combined HIV and maternal and child health promotion in Iringa, Tanzania. Health Policy Plan. 2018;33(10):1096-1106. doi:10.1093/heapol/czy104

48. Corley AG, Thornton CP, Glass NE. The Role of Nurses and Community Health Workers in Confronting Neglected Tropical Diseases in SubSaharan Africa: A Systematic Review. PLoS Negl Trop Dis. 2016;10(9):e0004914. doi:10.1371/journal.pntd.0 $\underline{004914}$
49. Kalyango JN, Alfven T, Peterson S, Mugenyi K, Karamagi C, Rutebemberwa E. Integrated community case management of malaria and pneumonia increases prompt and appropriate treatment for pneumonia symptoms in children under five years in Eastern Uganda. Malar J. 2013;12(1):340. doi:10.1186/ 1475-2875-12-340

50. Yeboah-Antwi K, Pilingana P, Macleod WB, et al. Community case management of fever due to malaria and pneumonia in children under five in Zambia: a cluster randomized controlled trial. PLoS Med. 2010;7(9):e1000340. doi:10.1371/journal.pmed.10003 $\underline{40}$

51. Whittaker MA, Dean AJ, Chancellor A. Advocating for malaria elimination - learning from the successes of other infectious disease elimination programmes. Malar J. 2014;13(1):221. doi:10.1186/1475-2875-13-2 21

52. Nájera JA, González-Silva M, Alonso PL. Some lessons for the future from the Global Malaria Eradication Programme (1955-1969). PLoS Med. 2011;8(1):e1000412. doi:10.1371/journal.pmed.10004 $\underline{12}$

53. Wilson J. The ethics of disease eradication. Vaccine. 2014;32(52):7179-7183. doi:10.1016/j.vaccin e.2014.10.009

54. Whitty CJM. Political, social and technical risks in the last stages of disease eradication campaigns. Int Health. 2015;7(5):302-303. doi:10.1093/inthealth/ihv 049 